\title{
Key Collisions of the RC4 Stream Cipher
}

\author{
Mitsuru Matsui \\ Information Technology R\&D Center \\ Mitsubishi Electric Corporation \\ 5-1-1 Ofuna Kamakura Kanagawa 247-8501, Japan \\ Matsui.Mitsuru@ab.MitsubishiElectric.co.jp
}

\begin{abstract}
This paper studies "colliding keys" of RC4 that create the same initial state and hence generate the same pseudo-random byte stream. It is easy to see that RC4 has colliding keys when its key size is very large, but it was unknown whether such key collisions exist for shorter key sizes. We present a new state transition sequence of the key scheduling algorithm for a related key pair of an arbitrary fixed length that can lead to key collisions and show as an example a 24-byte colliding key pair. We also demonstrate that it is very likely that RC4 has a colliding key pair even if its key size is less than 20 bytes. This result is remarkable in that the number of possible initial states of $\mathrm{RC} 4$ reaches $256 ! \approx 2^{1684}$. In addition we present a 20 -byte near-colliding key pair whose 256 -byte initial state arrays differ at only two byte positions.
\end{abstract}

\section{Introduction}

The RC4 stream cipher is one of the most widely-used real-world cryptosystems. Since its development in 1980's, RC4 has been used in various software applications and standard protocols such as Microsoft Office, Secure Socket Layer (SSL), Wired Equivalent Privacy (WEP). The architecture of RC4 is extremely simple and its encryption speed is remarkably fast. It has been suitable for not only $\mathrm{PC}$ applications but also embedding environments.

RC4 accepts a secret key whose length is 1 to 256 bytes, where a typical key size is 5 bytes (due to an old export regulation), 13 bytes (in the WEP encryption) or 16 bytes. It consists of two parts; the key scheduling algorithm (KSA) and the pseudo-random generating algorithm (PRGA). The KSA creates the 256-byte initial state from the secret key, and the PRGA generates pseudo-random byte stream from the initial state. Either of the algorithms can be described unambiguously in only a few lines. Due to this simplicity and wide applicability, a vast amount of efforts have been made for cryptanalysing RC4 since its specification was made public on Internet in 1994 [1].

As early as one year after the specification of RC4 was publicly available, it was pointed out by Roos [10] that the initial few bytes of its output stream are strongly correlated with the key. This observation was later extended in various ways. Mantin and Shamir [8] mounted a distinguishing attack of RC4 under the "strong distinguisher scenario" that an attacker can obtain many 
short output streams using random and unrelated keys. Paul and Preneel [9] successfully demonstrated a distinguisher that requires a total of $2^{25}$ output bytes ( 2 bytes/key $\times 2^{24}$ keys).

For cryptanalysis of a real-world application using RC4, Fluhrer, Mantin and Shamir [3] showed an attack of WEP for the first time in 2001. They found that partial information of the key of RC4 gives non-negligible information of its output bytes. This fact was efficiently used for mounting a passive attack, since the initial vector of WEP is packet-variable and embedded in the key of RC4. It was recently improved by Klein 6, and finally in 2007 Tews, Weinmann and Pyskin [11] and Vaudenay and Vuagnoux [12 independently demonstrated a very practical key recovery attack of the 104-bit WEP.

For another attacks, Fluhrer and McGrew 4] presented a distinguishing attack of RC4 using $2^{30.6}$ output bytes generated by a single key, and Mantin [7] successfully reduced the output stream size required for the successful attack down to $2^{26.5}$ bytes and also showed how to predict output bits. More recently Biham and Carmeli 2] concentrated on the key scheduling algorithm of RC4 and discussed how to recover a secret key from a given internal state.

This paper studies another type of weakness of the key scheduling algorithm of RC4; that is, existence of secret keys that create the same initial state and hence generate the same pseudo-random byte stream, which we call "colliding keys". It had been already pointed out by Grosul and Wallach [5] in 2000 that RC4 has related-key key pairs that generate substantially similar hundred output bytes when the key size is close to the full 256 bytes. In this paper we explore much stronger key collisions in a shorter key size.

Since the total number of possible initial states of $\mathrm{RC} 4$ reaches $256 ! \approx 2^{1684}$, RC4 must have colliding keys if its key size exceeds $\lfloor 1684 / 8\rfloor=210$ bytes. Moreover, due to the birthday paradox, it is not surprising that RC4 has colliding keys when its key size is $\lfloor(1684 / 2) / 8\rfloor=105$ (or more) bytes. However, it was unknown whether colliding keys exist in a shorter key size. The contribution of this paper is to give a positive answer to this problem.

In this paper, we begin by demonstrating a specific example of a colliding 64-byte key pair, whose internal 256-byte state arrays differ at most two byte positions in any step $i(0 \leq i \leq 255)$ of the key scheduling algorithm. Then by generalizing this example, we show a state transition pattern that is applicable to a key pair of an arbitrary fixed length and estimate the probability that such pattern actually takes place for randomly given key pairs with a fixed difference.

We have confirmed that our probability estimation mostly agrees with computer experimental results when the key size is around 32 bytes or more. We also demonstrate that it is very likely that RC4 has a colliding key pair even when its key size is much shorter, say 20 bytes, while the minimal key size that has colliding keys is still an open problem.

We further extend our observation to a near-colliding key pair; that is, a key pair whose initial states differ at exactly two positions. In the same way as the key collision case, we show a state transition pattern of a key pair of an arbitrary length that can lead to a near-collision and analyze the probability 
that the pattern actually takes place. Finally we illustrate our (near-)colliding key pair search algorithm, which has successfully found a 24-byte colliding key pair and a 20-byte near-colliding key pair.

\section{The RC4 Stream Cipher}

$\mathrm{RC} 4$ is a stream cipher with a secret key whose length is 1 to 256 bytes. We define $\mathrm{k}$ and $\mathrm{K}$ as the target key size in byte and the $\mathrm{k}$-byte secret key, respectively, and K [0] ... K[k-1] denote k key bytes. For arbitrary i, K [i] means K [i mod k] . $\mathrm{RC} 4$ consists of the key scheduling algorithm (KSA), which creates the 256-byte initial state array $\mathrm{S}[0] \ldots \mathrm{S}[255]$ from the secret key, and the pseudo-random generating algorithm (PRGA), which generates byte sequence Z [0] ... Z [L-1] of arbitrary length L from the initial state.

This paper discusses colliding key pairs of RC4 that create the same initial state. Hence only the key scheduling algorithm, described below in the syntax of $\mathrm{C}$ language, is relevant. When necessary, we use notations $\mathrm{S} 1 / \mathrm{S} 2$ and $j 1 / j 2$ for the state array and the state index for the first and second key K1/K2, respectively. The goal of this paper is to find, or show a strong evidence of existence of, key pair K1 and K2 such that the corresponding state arrays S1 and S2 are completely same at the end of the key scheduling algorithm.

We define "the distance of a key pair at step $i$ " as the number of distinct bytes between S1 and S2 at the bottom (i.e. after the swap) of the i-th iteration in the state randomization loop. If the distance of key pair K1 and K2 is 0 at step 255, then they are a colliding key pair. This paper will deal with key pairs whose distance is at most 2 at any step $i(0 \leq i \leq 255)$.

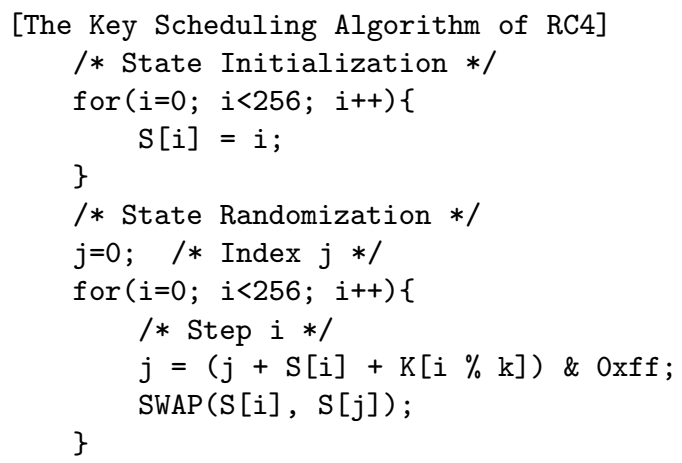

[The Pseudo-Random Generator Algorithm of RC4]

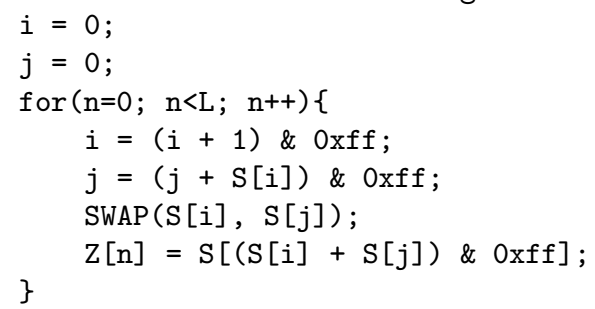




\section{An Example: How It Works}

In this section we demonstrate a specific example of a colliding key pair and explain how its collision is created in a step-by-step fashion. In fact, this simple example contains all tricks that we need for finding colliding key pairs of an arbitrary length in later sections. The following is a 64-byte key pair, written in hexadecimal form, which differs at only one byte position, $\mathrm{K} 1[2] \neq \mathrm{K} 2[2]$. These two keys create the same initial state, and hence they are cryptographically indistinguishable.

$$
\begin{aligned}
& \mathrm{K} 1=45 \quad 3 d \quad 7 d \quad 3 d \quad c 945 \quad 57 \quad 1200 \quad 0000 \quad 000000 \quad 00 \quad 00 \\
& \begin{array}{llllllllllllllll}
00 & 00 & 00 & 00 & 00 & 00 & 00 & 00 & 00 & 00 & 00 & 00 & 00 & 00 & 00 & 00
\end{array} \\
& \begin{array}{llllllllllllllll}
00 & 00 & 00 & 00 & 00 & 00 & 00 & 00 & 00 & 00 & 00 & 00 & 00 & 00 & 00 & 00
\end{array} \\
& \begin{array}{llllllllllllllll}
00 & 00 & 00 & 00 & 00 & 00 & 00 & 00 & 00 & 00 & 00 & 00 & 00 & 00 & 00
\end{array} \\
& \mathrm{~K} 2=45 \quad 3 d \quad 7 e \quad 3 d \quad c 945 \quad 57 \quad 1200 \quad 00 \quad 00 \quad 000000 \quad 00 \quad 00 \\
& \begin{array}{llllllllllllllll}
00 & 00 & 00 & 00 & 00 & 00 & 00 & 00 & 00 & 00 & 00 & 00 & 00 & 00 & 00 & 00
\end{array} \\
& \begin{array}{llllllllllllllll}
00 & 00 & 00 & 00 & 00 & 00 & 00 & 00 & 00 & 00 & 00 & 00 & 00 & 00 & 00 & 00
\end{array} \\
& \begin{array}{llllllllllllllll}
00 & 00 & 00 & 00 & 00 & 00 & 00 & 00 & 00 & 00 & 00 & 00 & 00 & 00 & 00 & 00
\end{array}
\end{aligned}
$$

\begin{tabular}{|c|c|c|c|}
\hline$i$ & K1 K2 & $j 1 j 2$ & differences between S1 and S2 \\
\hline 00 & 4545 & 4545 & $\mathrm{~S} 1=\mathrm{S} 2$ \\
\hline 01 & $3 d 3 d$ & 8383 & $\mathrm{~S} 1=\mathrm{S} 2$ \\
\hline 02 & $7 d 7 e$ & 0203 & $@ 02(\mathrm{~S} 1=02 \quad \mathrm{~S} 2=03)$ \\
\hline 03 & $3 d \quad 3 d$ & 4242 & $@ 02(\mathrm{~S} 1=02 \quad \mathrm{~S} 2=03) \quad @ 42(\mathrm{~S} 1=03 \quad \mathrm{~S} 2=02)$ \\
\hline $04-40$ & & & $@ 02(\mathrm{~S} 1=02 \mathrm{~S} 2=03) \quad @ 42(\mathrm{~S} 1=03 \mathrm{~S} 2=02)$ \\
\hline 41 & $3 d 3 d$ & 0202 & $@ 41(\mathrm{~S} 1=02 \mathrm{~S} 2=03) \quad @ 42(\mathrm{~S} 1=03 \quad \mathrm{~S} 2=02)$ \\
\hline 42 & $7 \mathrm{~d} 7 \mathrm{e}$ & 8282 & $@ 41(\mathrm{~S} 1=02 \mathrm{~S} 2=03) \quad @ 82(\mathrm{~S} 1=03 \mathrm{~S} 2=02)$ \\
\hline $43-80$ & & & $@ 41(\mathrm{~S} 1=02 \mathrm{~S} 2=03) \quad @ 82(\mathrm{~S} 1=03 \mathrm{~S} 2=02)$ \\
\hline 81 & $3 d 3 d$ & 4141 & $@ 81(\mathrm{~S} 1=02 \mathrm{~S} 2=03) \quad$ (82 $(\mathrm{S} 1=03 \mathrm{~S} 2=02)$ \\
\hline 82 & $7 d 7 e$ & c1 c1 & @81 (S1=02 S2=03) @c1(S1=03 S2=02) \\
\hline $83-b f$ & & & @81 (S1=02 S2=03) @c1 (S1=03 S2=02) \\
\hline $\mathrm{c0}$ & 4545 & 8181 & $@ \mathrm{c} 0(\mathrm{~S} 1=02 \mathrm{~S} 2=03) \quad @ \mathrm{c} 1(\mathrm{~S} 1=03 \mathrm{~S} 2=02)$ \\
\hline$c 1$ & $3 d 3 d$ & c1 c0 & $\mathrm{S} 1=\mathrm{S} 2$ \\
\hline c2 & $7 \mathrm{~d} 7 \mathrm{e}$ & 5454 & $\mathrm{~S} 1=\mathrm{S} 2$ \\
\hline$c 3-f f$ & & & $\mathrm{~S} 1=\mathrm{S} 2$ \\
\hline
\end{tabular}

Table 1 shows internal values in the state randomization loop for each key, where "@xX" denotes an address of the state array. For example, at the end of step $i=02$ (i.e. after the swap), $j 1=02, j 2=03$ and the state arrays differ at exactly two positions 02 and 03. At these positions, S1 [02] =02, S2 [02]=03, S1 [03] =03 and S2 [03] $=02$.

Table 1. The State Transition Pattern of the 64-byte Key Pair

Now let us take a look at this transition sequence more closely to see why/how the distance of this key pair remains 0 or 2 at any step. 
Table 2. The State Transition Details of the 64-bit Key Pair

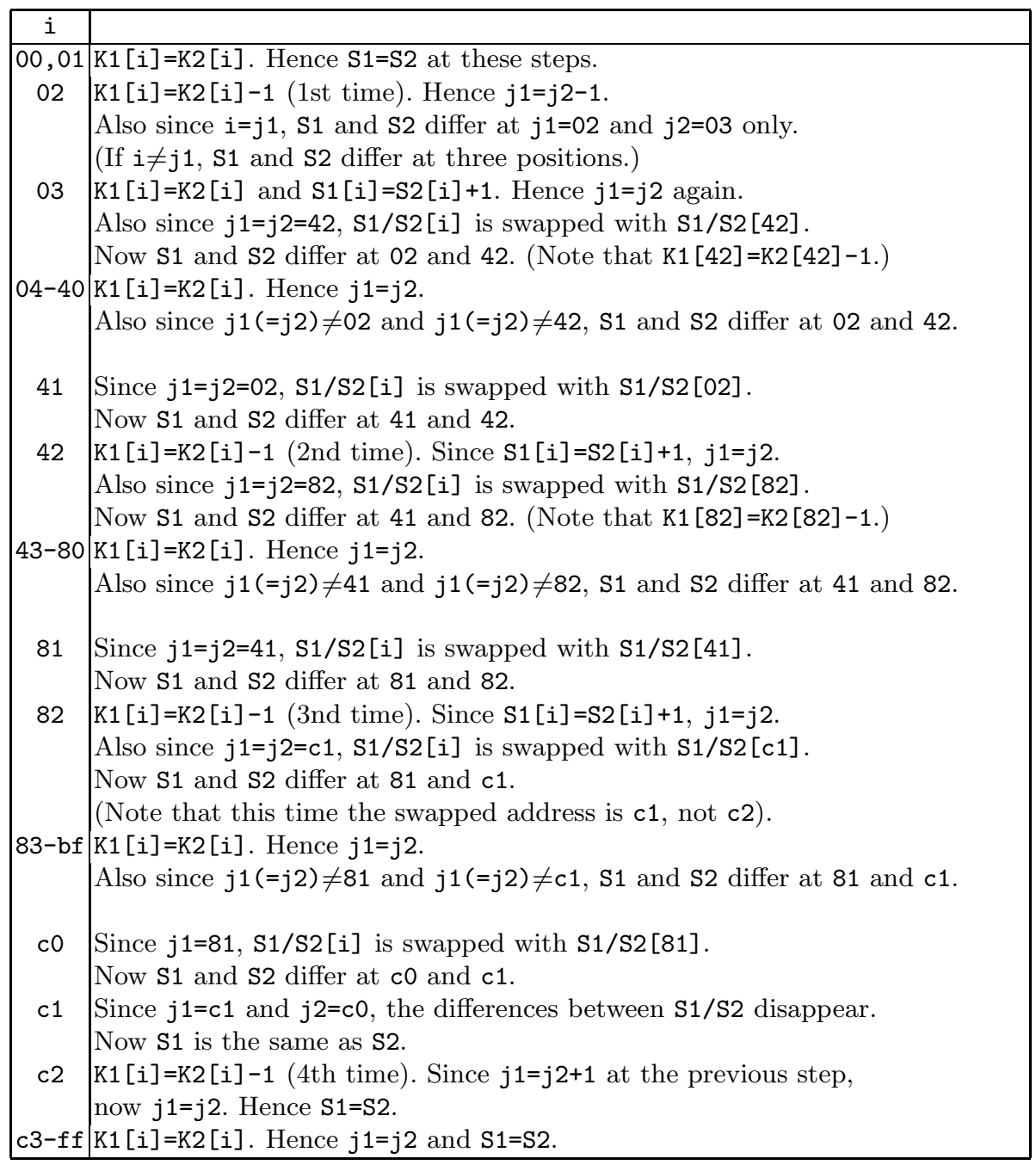

We have $\mathrm{K} 1[\mathrm{i}]=\mathrm{K} 2[\mathrm{i}]-1$ four times. For $i=02$, this difference is absorbed at the next step because it causes $j 1=j 2-1$ while $S 1[3]=S 2[3]+1$. Note that the relation $\mathrm{K} 1[2]=\mathrm{K} 2[2]-1$ is essential for this example. For $i=42$ and $i=82, j 1$ remains the same as $\mathrm{j} 2$ because $\mathrm{S} 1$ [42]=S2 [42]+1 and $\mathrm{S} 1$ [82]=S2 [82]+1, respectively.

The last time $i=c 2$ is a bit tricky; at two steps before $i=c 0, S 1$ and $S 2$ differ at $c 0$ and $c 1$. Moreover at $i=c 1, j 1=c 0$ and $j 2=c 1$ and hence the differences of $\mathrm{S} 1$ and $\mathrm{S} 2$ disappear at this step. The remaining difference is between $\mathrm{j} 1$ and $\mathrm{j} 2$, which is finally cancelled at $\mathrm{i}=\mathrm{c} 2$ due to the relation $\mathrm{K} 1$ [c2] =K2 [c2]-1. 


\section{General Collision Sequence}

In this section we extend the sequence shown in the previous section to a key pair of an arbitrary length and give a general transition pattern that can lead to the same initial state. We also estimate the probability that the key collision actually occurs for randomly given key pairs with a fixed difference.

We here consider k-byte key pair K1 and K2 such that K1 [i] $=K 2[i]-1$ if $i=d$ $(0 \leq \mathrm{d}<\mathrm{k})$ and $\mathrm{K} 1[\mathrm{i}]=\mathrm{K} 2[\mathrm{i}]$ otherwise. We also define $\mathrm{n}$ as $|(256+\mathrm{k}-1-\mathrm{d}) / \mathrm{k}|$. Then $\mathrm{K} 1[\mathrm{i}] \neq \mathrm{K} 2[\mathrm{i}]$ takes place exactly $\mathrm{n}$ times in the state randomization loop.

Table 3 illustrates details of the state transition pattern for given $\mathrm{k}$ and $\mathrm{d}$ with estimated probability, where "-" allows any address (a "don't-care" value) and " $\mathrm{e}$ " at step $\mathrm{i}=\mathrm{d}+(\mathrm{n}-1) \mathrm{k}-2$ is the value such that $\mathrm{S} 1[\mathrm{x}]=\mathrm{d}$ and $\mathrm{S} 2[\mathrm{x}]=\mathrm{d}+1$ at step $i=d+(n-1) k-3$.

Table 3. The State Transition Pattern of a k-byte Colliding Key Pair

\begin{tabular}{|c|c|c|c|}
\hline & Step & Internal State Values & Estimated Prob. \\
\hline & $i=0 \ldots d-1$ & $\begin{array}{l}j 1(=j 2) \neq d, \quad j 1(=j 2) \neq d+1 \\
S 1=S 2\end{array}$ & $\begin{array}{l}(254 / 256)^{d} \text { or } \\
(254 / 256)^{d-1}\end{array}$ \\
\hline (b) & $i=d$ & $\begin{array}{l}j 1=d, j 2=d+1 \\
Q d(S 1=d \quad S 2=d+1)\end{array}$ & $1 / 256$ \\
\hline (c) & $i=d+1$ & $\begin{array}{l}\mathrm{j} 1=\mathrm{j} 2=\mathrm{d}+\mathrm{k} \\
@ \mathrm{~d}(\mathrm{~S} 1=\mathrm{d} \quad \mathrm{S} 2=\mathrm{d}+1) \\
\bigotimes d+\mathrm{k}(\mathrm{S} 1=\mathrm{d}+1 \quad \mathrm{~S} 2=\mathrm{d})\end{array}$ & $1 / 256$ \\
\hline (d) & $i=d+2 \ldots d+k-1$ & $\begin{array}{l}\mathrm{j} 1(=\mathrm{j} 2) \neq \mathrm{d}+\mathrm{k} \\
\bigotimes-(\mathrm{S} 1=\mathrm{d} \quad \mathrm{S} 2=\mathrm{d}+1) \\
@ \mathrm{~d}+\mathrm{k}(\mathrm{S} 1=\mathrm{d}+1 \quad \mathrm{~S} 2=\mathrm{d})\end{array}$ & $(255 / 256)^{k-2}$ \\
\hline
\end{tabular}

Repeat steps (e) and (f) for $\mathrm{m}=1 \ldots \mathrm{n}-3$.

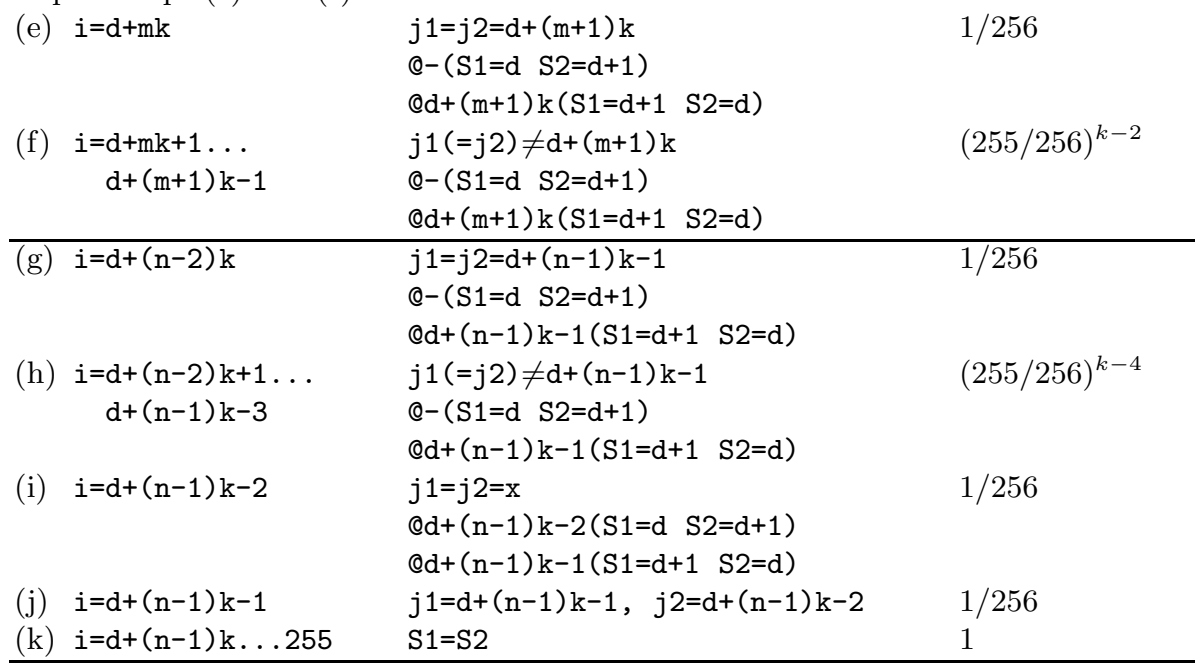


The probability that event (a) takes place is $(254 / 256)^{d-1}$ only if $\mathrm{d}=\mathrm{k}-1$, and $(254 / 256)^{d}$ otherwise, because K1 [0] =K2 [0] =0 when $\mathrm{d}=\mathrm{k}-1$ (also see below). Now assuming that the state index $\mathrm{j}$ is uniformly random at all steps, the probability that this transition sequence actually takes place for randomly given key pairs with the fixed difference is

$\operatorname{ColProb}(k, d)=\left\{\begin{array}{ll}(254 / 256)^{d}(255 / 256)^{(n-1)(k-2)-2}(1 / 256)^{n+2} & (d \neq k-1) \\ (254 / 256)^{d-1}(255 / 256)^{(n-1)(k-2)-2}(1 / 256)^{n+2} & (d=k-1)\end{array}\right.$.

This probability is actually very close to $1 / e(1 / 256)^{n+2}$, which depends on $n$ only. Of course $j$ 's are not uniformly random in practice, and therefore this probability estimation is not necessarily correct. However this (very intuitive) assumption mostly agrees with our computer experimental results when the length of the key is around 32 bytes or more.

The following is one of the 43-byte colliding key pairs that we found (43 is the minimal $\mathrm{k}$ such that $\lfloor 256 / \mathrm{k}\rfloor=5$ ), and its experimentally observed conditional probability of each event. These keys differ at the last byte, hence $d=42$. Note that successful events (a) and (b) effectively determine one-byte information of the key. In other words, $\mathrm{K}[0] \ldots \mathrm{K}[\mathrm{d}-1]$ uniquely determines $\mathrm{K}[\mathrm{d}]$. Moreover for meeting events (b) and (c), we must have $\mathrm{K} 1[0]=\mathrm{K} 2[0]=0$, more generally $\mathrm{K} 1[\mathrm{~d}+1]=\mathrm{K} 2[\mathrm{~d}+1]=\mathrm{k}-\mathrm{d}-1$. We hence do not have to "wait for" events (b) and (c) in searching for key collisions.

Table 4 shows that our experimental results, where we obtained two 43-byte colliding key pairs from $2^{41.5}$ candidate pairs, perfectly agree with our probability estimation.

Table 4. Experimental Results of Finding 43-byte Colliding Key Pairs

\begin{tabular}{lll}
\hline Event & Estimated Prob. & Measured Prob. \\
\hline (a) & $0.725010=(254 / 256)^{41}$ & $0.725010=2272363208729 / 3134252384256$ \\
(d) & $0.851743=(255 / 256)^{41}$ & $0.851638=1935231636873 / 2272363208729$ \\
(e- 1$)$ & $0.003906=1 / 256$ & $0.004120=7973306038 / 1935231636873$ \\
(f-1) & $0.851743=(255 / 256)^{41}$ & $0.851706=6790914484 / 7973306038$ \\
(e-2) & $0.003906=1 / 256$ & $0.003933=26707884 / 6790914484$ \\
(f- $)$ & $0.851743=(255 / 256)^{41}$ & $0.851605=22744564 / 26707884$ \\
(g) & $0.003906=1 / 256$ & $0.003891=88498 / 22744564$ \\
(h) & $0.858437=(255 / 256)^{39}$ & $0.858336=75961 / 88498$ \\
(i) & $0.003906=1 / 256$ & $0.003976=302 / 75961$ \\
(j) & $0.003906=1 / 256$ & $0.006623=2 / 302$ \\
\hline
\end{tabular}

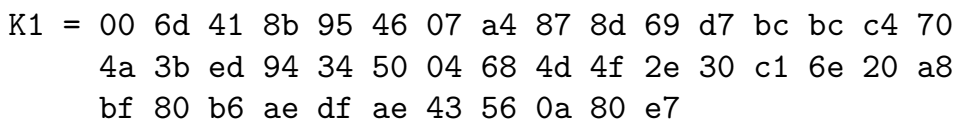


Also, assuming again that $\operatorname{ColProb}(k, d)$ is correct for any $\mathrm{k}$ and $\mathrm{d}$, the expected number of $\mathrm{k}$-byte colliding key pairs out of a total of $2^{8 k}$ keys is

$$
\operatorname{ColPairs}(k)=2^{8 k} \times \sum_{d=0}^{k-1} \operatorname{ColProb}(k, d) \text {. }
$$

Table 5 is a list of $\log _{2}(\operatorname{ColPairs}(k))$ for $\mathrm{k}=17 \ldots 64$. This table clearly shows that key collisions can exist in much shorter key length. On the other hand, $\operatorname{ColProb}(k, d)$ does not always agree with our experimental results when $\mathrm{k}$ is small, say 30 or less, because probabilistic dependency of j's cannot be ignored in such range. It seems that more detailed probability analysis is needed for accurately estimating the density of colliding key pairs in a small key size.

Table 5. List of $\log _{2}(\operatorname{ColPairs}(k))$ for $\mathrm{k}=17 \ldots 64$

\begin{tabular}{|cl|cl|rl|rl|rl|}
\hline $\mathrm{k}$ & Pairs & $\mathrm{k}$ & Pairs & $\mathrm{k}$ & Pairs & $\mathrm{k}$ & Pairs & $\mathrm{k}$ & Pairs \\
\hline 15 & - & 25 & 106.9 & 35 & 211.2 & 45 & 306.4 & 55 & 394.9 \\
16 & - & 26 & 120.7 & 36 & 219.6 & 46 & 314.9 & 56 & 403.2 \\
17 & 2.7 & 27 & 130.5 & 37 & 232.3 & 47 & 323.3 & 57 & 411.5 \\
18 & 18.5 & 28 & 139.2 & 38 & 242.0 & 48 & 331.6 & 58 & 419.7 \\
19 & 34.0 & 29 & 153.0 & 39 & 250.7 & 49 & 339.9 & 59 & 427.9 \\
20 & 48.7 & 30 & 162.5 & 40 & 259.2 & 50 & 348.1 & 60 & 436.1 \\
21 & 58.8 & 31 & 171.2 & 41 & 267.6 & 51 & 356.3 & 61 & 444.2 \\
22 & 73.7 & 32 & 179.7 & 42 & 275.9 & 52 & 368.7 & 62 & 452.4 \\
23 & 83.0 & 33 & 193.7 & 43 & 287.7 & 53 & 377.8 & 63 & 460.5 \\
24 & 97.7 & 34 & 202.6 & 44 & 297.6 & 54 & 386.4 & 64 & 468.6 \\
\hline
\end{tabular}

\section{Near-Collision Sequence}

This section gives another extension of section 2, a near-colliding key pair whose initial state arrays S1 and S2 differ at exactly two positions. We use the same notations as in the previous section.

Table 6 shows the details of our state transition pattern that can lead to a near-collision, which is the same as the collision case except the last part. "-" allows any address and " $x$ " is any value equal to or less than $d+(n-1) k$. Note that if $\mathrm{x}$ exceeds $\mathrm{d}+(\mathrm{n}-1) \mathrm{k}$, the distance between $\mathrm{S} 1$ and $\mathrm{S} 2$ exceeds two in $(\mathrm{h})$.

The expected probability that this transition pattern actually takes place is

$$
\begin{aligned}
& \text { NearColProb }(k, d)= \\
& \left\{\begin{array}{l}
(254 / 256)^{d}(255 / 256)^{(n-1)(k-2)}(1 / 256)^{n}(d+(n-1) k+1) / 256 \quad(d \neq k-1) \\
(254 / 256)^{d-1}(255 / 256)^{(n-1)(k-2)}(1 / 256)^{n}(d+(n-1) k+1) / 256(d=k-1)
\end{array}\right.
\end{aligned}
$$

This probability is actually very close to $1 / e(1 / 256)^{n}$, which depends on $n$ only, and hence roughly it holds that $N$ earColProb $(k, d)=2^{16} \operatorname{ColProb}(k, d)$. 
Table 6. The State Transition Pattern of a k-byte Near-Colliding Key Pair

\begin{tabular}{|c|c|c|c|}
\hline & Step & Internal State Values & Approx. Prob. \\
\hline & $i=0 \ldots d-1$ & $\begin{array}{l}j 1(=j 2) \neq d, \quad j 1(=j 2) \neq d+1 \\
S 1=S 2\end{array}$ & $\begin{array}{l}(254 / 256)^{d} \text { or } \\
(254 / 256)^{d-1}\end{array}$ \\
\hline (b) & $i=d$ & $\begin{array}{l}j 1=d, \quad j 2=d+1 \\
@ d(S 1=d \quad S 2=d+1) \\
@ d+1(S 1=d+1 \quad S 2=d)\end{array}$ & $1 / 256$ \\
\hline (c) & $i=d+1$ & $\begin{array}{l}j 1=j 2=d+k \\
@ d(S 1=d \quad S 2=d+1) \\
@ d+k(S 1=d+1 \quad S 2=d)\end{array}$ & $1 / 256$ \\
\hline (d) & $i=d+2 \ldots d+k-1$ & $\begin{array}{l}j 1(=j 2) \neq d+k \\
@-(S 1=d \quad S 2=d+1) \\
@ d+k(S 1=d+1 \quad S 2=d)\end{array}$ & $(255 / 256)^{k-2}$ \\
\hline
\end{tabular}

\begin{tabular}{|c|c|c|}
\hline \multicolumn{3}{|c|}{ Repeat steps (e) and (f) for $m=1 \ldots n-2$} \\
\hline \multirow[t]{3}{*}{ (e) $i=d+m k$} & $j 1=j 2=d+(m+1) k$ & $1 / 256$ \\
\hline & $@-(\mathrm{S} 1=\mathrm{d} \quad \mathrm{S} 2=\mathrm{d}+1)$ & \\
\hline & $@ d+(m+1) k(S 1=d+1 \quad S 2=d)$ & \\
\hline$i=d+m k+1 \ldots$ & $\mathrm{j} 1(=\mathrm{j} 2) \neq \mathrm{d}+(\mathrm{m}+1) \mathrm{k}$ & $(255 / 256)^{k-2}$ \\
\hline $\mathrm{d}+(\mathrm{m}+1) \mathrm{k}-1$ & $Q-(\mathrm{S} 1=\mathrm{d} \quad \mathrm{S} 2=\mathrm{d}+1)$ & \\
\hline & $@ d+(m+1) k(S 1=d+1 \quad S 2=d)$ & \\
\hline (g) $i=d+(n-1) k$ & $j 1=x, \quad j 2=x$ & $(\mathrm{~d}+(\mathrm{n}-1) \mathrm{k}+1) / 256$ \\
\hline & $Q-(\mathrm{S} 1=\mathrm{d} \quad \mathrm{S} 2=\mathrm{d}+1)$ & \\
\hline & $Q x(S 1=d+1 \quad S 2=d)$ & \\
\hline (h) $i=d+(n-1) k+1 \ldots 255$ & $Q-(\mathrm{S} 1=\mathrm{d} \quad \mathrm{S} 2=\mathrm{d}+1)$ & 1 \\
\hline & $Q-(\mathrm{S} 1=\mathrm{d}+1 \quad \mathrm{~S} 2=\mathrm{d})$ & \\
\hline
\end{tabular}

The following is a 33-byte near-colliding key pair that we found (33 is the minimal $\mathrm{k}$ such that $\lfloor 256 / \mathrm{k}\rfloor=7$ ), and its experimentally observed conditional probability of each event. These two keys differ at the last byte, hence $d=32$.

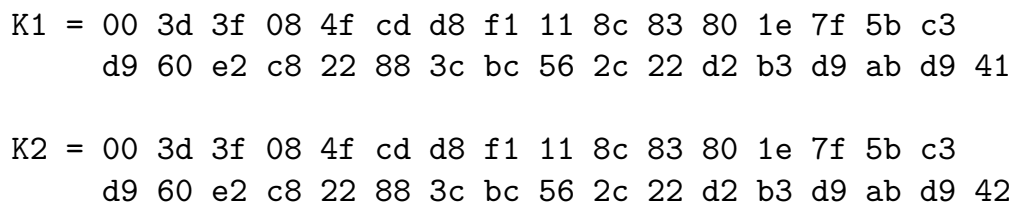

Table 7 shows that our experimental results, where we obtained four 33-byte near-colliding key pairs from $2^{44.2}$ candidates, mostly agree with our probability estimation. Now the expected number of $k$-byte near-colliding key pairs out of a total of $2^{8 k}$ keys is

$$
\operatorname{NearColPairs}(k)=2^{8 k} \times \sum_{d=0}^{k-1} N \text { earColProb }(k, d) \text {. }
$$

Table 8 is a list of $\log _{2}(N$ earColPairs $(k))$ for $k=16 \ldots 64$. This table clearly shows that near-key collisions can also exist in much shorter key length. However, 
Table 7. Experimental Results of Finding 33-byte Near-colliding Key Pairs

\begin{tabular}{lll}
\hline Event & Estimated Prob. & Measured Prob. \\
\hline (a) & $0.784163=(254 / 256)^{31}$ & $0.784163=15852958662942 / 20216411062272$ \\
(d) & $0.885741=(255 / 256)^{31}$ & $0.885471=14037333620475 / 15852958662942$ \\
(e-1) & $0.003906=1 / 256$ & $0.001946=27312181761 / 14037333620475$ \\
(f-1) & $0.885741=(255 / 256)^{31}$ & $0.885555=24186440984 / 27312181761$ \\
(e-2) & $0.003906=1 / 256$ & $0.003954=95628579 / 24186440984$ \\
(f-2) & $0.885741=(255 / 256)^{31}$ & $0.885499=84679053 / 95628579$ \\
(e-3) & $0.003906=1 / 256$ & $0.003930=332809 / 84679053$ \\
(f-3) & $0.885741=(255 / 256)^{31}$ & $0.886160=885741 / 332809$ \\
(e-4) & $0.003906=1 / 256$ & $0.003774=1113 / 294922$ \\
(f-4) & $0.882281=(255 / 256)^{31}$ & $0.881402=981 / 1113$ \\
(e-5) & $0.003906=1 / 256$ & $0.005097=5 / 981$ \\
(f-5) & $0.882281=(255 / 256)^{31}$ & $0.800000=4 / 5$ \\
(g) & $0.902344=231 / 256$ & $1.000000=4 / 4$ \\
\hline
\end{tabular}

Table 8. List of $\log _{2}($ NearColPairs $(k))$ for $\mathrm{k}=16 \ldots 64$

\begin{tabular}{|cl|cl|cc|cc|cc|}
\hline $\mathrm{k}$ & Pairs & $\mathrm{k}$ & Pairs & $\mathrm{k}$ & Pairs & $\mathrm{k}$ & Pairs & $\mathrm{k}$ & Pairs \\
\hline 15 & - & 25 & 122.8 & 35 & 227.1 & 45 & 322.2 & 55 & 410.6 \\
16 & 2.7 & 26 & 136.5 & 36 & 235.5 & 46 & 330.7 & 56 & 418.9 \\
17 & 18.7 & 27 & 146.3 & 37 & 248.1 & 47 & 339.1 & 57 & 427.2 \\
18 & 34.5 & 28 & 155.1 & 38 & 257.8 & 48 & 347.4 & 58 & 435.4 \\
19 & 49.9 & 29 & 168.8 & 39 & 266.5 & 49 & 355.7 & 59 & 443.6 \\
20 & 64.6 & 30 & 178.3 & 40 & 275.0 & 50 & 363.9 & 60 & 451.8 \\
21 & 74.7 & 31 & 187.1 & 41 & 283.4 & 51 & 372.1 & 61 & 460.0 \\
22 & 89.6 & 32 & 195.5 & 42 & 291.7 & 52 & 384.4 & 62 & 468.1 \\
23 & 98.9 & 33 & 209.5 & 43 & 303.5 & 53 & 393.5 & 63 & 476.3 \\
24 & 113.6 & 34 & 218.5 & 44 & 313.4 & 54 & 402.1 & 64 & 484.4 \\
\hline
\end{tabular}

again, more detailed probabilistic analysis is needed for accurately estimating the density of near-colliding key pairs in a small key size.

\section{Faster Collision Search}

In previous sections we searched for colliding and near-colliding key pairs in a very simple fashion, - checking transition patterns step-by-step and if fails, restarting the search with another random candidate - , whose primary purpose was to confirm theoretical claims experimentally. In this section we explore a faster method for finding (near-)colliding key pairs for smaller key sizes.

We here try to find (near-)colliding key pairs by checking distance between two keys at each step but not checking our transition patters. We now define MaxColStep $(K 1, K 2)$ as maximal step $i$ such that distance between $K 1$ and $K 2$ is at most two at all steps up to step $i$. For a colliding or near-colliding key 
pair, $\operatorname{MaxColStep}(K 1, K 2)=255$. Also for given key $K$ and $0 \leq x, y<256$, we define a slightly modified key $\mathrm{K}<\mathrm{x}, \mathrm{y}>$ as

$$
\begin{array}{ll}
K<x, y>[x] & =K[x]+y \\
K<x, y>[x+1] & =K[x+1]-y \\
K<x, y>[i] & =K[i] \quad \text { if i is not } x \text { or } x+1
\end{array}
$$

It is naturally expected that the given key $\mathrm{K}$ and its modified keys $\mathrm{K}\langle\mathrm{x}, \mathrm{y}\rangle$ are likely to create similar initial states. Hence when the check fails for K1 and K2, we can try $\mathrm{K} 1<\mathrm{x}, \mathrm{y}\rangle$ and $\mathrm{K} 2<\mathrm{x}, \mathrm{y}\rangle$, which are likely to be a better pair, instead of rewinding and restarting the search with another random candidate. Which pair is better (i.e. closer to an actual (near-)collision) can be measured by the MaxColStep function. These observations lead to the following simple recursive search algorithm,

Collision Search Algorithm: Generate a random key pair K1 and K2 which differ at position $d$ by one. Set $K 1[d+1]=K 2[d+1]=k-d-1$ (see section 4$)$ and call $\operatorname{Search}(\mathrm{K} 1, \mathrm{~K} 2)$. Repeat this until a (near-)colliding key pair is found:

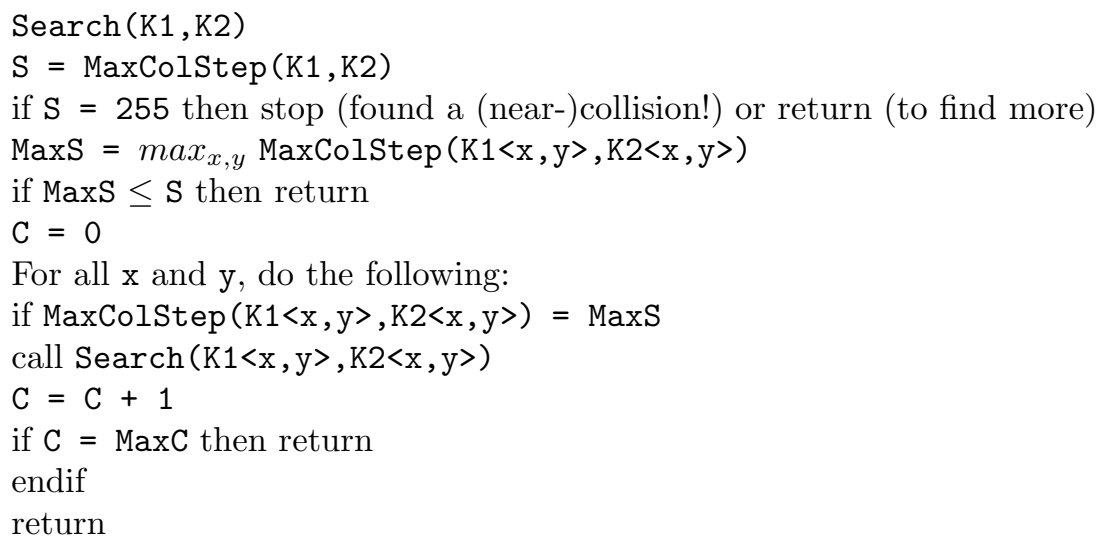

where $\mathrm{x}$ runs from 0 to 255 except $\mathrm{d}$ and $\mathrm{d}+1$ (for not changing $\mathrm{K} 1[\mathrm{~d}+1] / \mathrm{K} 2$ $[\mathrm{d}+1])$, and y runs from 1 to 255 . This algorithm finds mostly near-colliding key pairs, but may also find colliding key pairs (if we are lucky enough or patient enough). In fact, using this algorithm we reached a 24-byte colliding key pair and a 20-byte near-colliding key pair as follows:

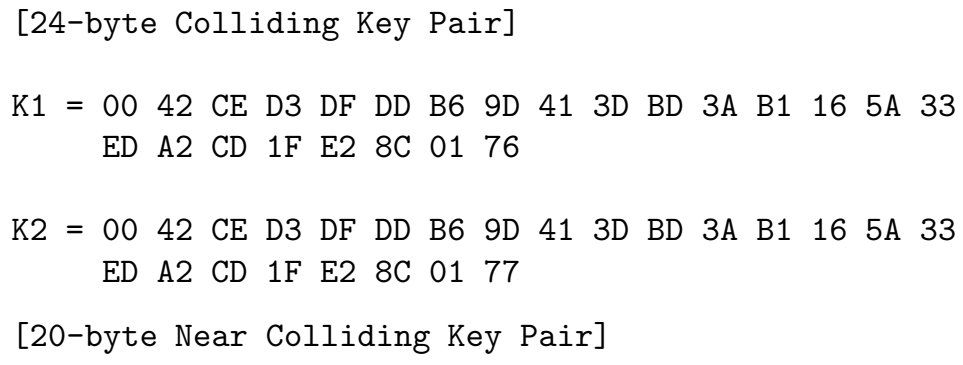


MaxC is a pre-defined value. In our experiments, the search worked efficiently when MaxC is around 10, and the maximal depth of recursive calls was less than 20. Obviously this algorithm has a room for improvement. For instance, it can pick up the same near-colliding key pair twice or more; that is, the search contains some redundancy. Also another evaluation function, instead of MaxColStep, or another key modification is a possibility. Studying a better collision search method seems an interesting future topic.

\section{Concluding Remarks}

This paper explored key collisions of the RC4 stream cipher. We presented a 24byte colliding key pair and a 20-byte near-colliding key pair, and demonstrated that our probabilistic analysis well agrees with experimental results when the key size is around 32 bytes. It seems now very likely that RC4 has colliding keys in even smaller key length, say less than 20 bytes.

While tables 5 and 8 suggest an existence of 17-byte colliding key pairs and 16-byte near-colliding key pairs, respectively, further research is needed for more accurately estimating $\operatorname{ColProb}(k, d)$ and $N$ earColProb $(k, d)$ in such small $k$. In fact, we have already seen that the observed probability of event (e-1) in table 7 was much smaller than the expected probability $1 / 256$. This kind of phenomenons (much larger or smaller than our estimation) frequently appears when $k$ is small.

It might not be very easy to derive a simple formula of $\operatorname{ColProb}(k, d)$ and NearColProb $(k, d)$ applicable to all $k$ and $d$. As far as we know, this is the first paper that went deep into key collisions of RC4. We hope that our observation will lead to further study of this direction.

\section{Acknowledgements}

The author would like to thank an anonymous reviewer for suggesting the fast search algorithm, which has been further improved by the author and included in section 6. Another anonymous reviewer has pointed out that $\operatorname{ColProb}(k, d)$ and $N$ earColProb $(k, d)$ can approximate to $1 / e(1 / 256)^{n+2}$ and $1 / e(1 / 256)^{n}$, respectively, which makes things much clearer. The author is grateful to all reviewers for their valuable and constructive comments.

\section{References}

1. Anonymous: RC4 Source Code. CypherPunks mailing list (September 9, 1994), http://cypherpunks.venona.com/date/1994/09/msg00304.html, http://groups.google.com/group/sci.crypt/msg/10a300c9d21afca0 
2. Biham, E., Carmeli, Y.: Efficient Reconstruction of RC4 Keys from Internal States. In: Nyberg, K. (ed.) FSE 2008. LNCS, vol. 5086, pp. 270-288. Springer, Heidelberg (2008)

3. Fluhrer, S., Mantin, I., Shamir, A.: Weaknesses in the Key Scheduling Algorithm of RC4. In: Vaudenay, S., Youssef, A.M. (eds.) SAC 2001. LNCS, vol. 2259, pp. 1-24. Springer, Heidelberg (2001)

4. Fluhrer, S., McGrew, D.: Statistical Analysis of the Alleged RC4 Keystream Generator. In: Schneier, B. (ed.) FSE 2000. LNCS, vol. 1978, pp. 19-30. Springer, Heidelberg (2000)

5. Grosul, A.L., Wallach, D.S.: A Related-Key Cryptanalysis of RC4. Technical Report TR-00-358, Department of Computer Science, Rice University (2000),

http://cohesion.rice.edu/engineering/computerscience/tr/ TR_Download.cfm?SDID $=126$

6. Klein, A.: Attacks on the RC4 Stream Cipher. Designs, Codes and Cryptography 48(3), 269-286 (2008)

7. Mantin, I.: Predicting and Distinguishing Attacks on RC4 Keystream Generator. In: Cramer, R. (ed.) EUROCRYPT 2005. LNCS, vol. 3494, pp. 491-506. Springer, Heidelberg (2005)

8. Mantin, I., Shamir, A.: A Practical Attack on Broadcast RC4. In: Matsui, M. (ed.) FSE 2001. LNCS, vol. 2355, pp. 152-164. Springer, Heidelberg (2001)

9. Paul, S., Preneel, B.: A New Weakness in the RC4 Keystream Generator and an Approach to Improve Security of the Cipher. In: Roy, B., Meier, W. (eds.) FSE 2004. LNCS, vol. 3017, pp. 245-259. Springer, Heidelberg (2004)

10. Roos, A.: A Class of Weak Keys in the RC4 Stream Cipher (1995), http://marcel.wanda.ch/Archive/WeakKeys

11. Tews, E., Weinmann, R.P., Pyshkin, A.: Breaking 104 Bit WEP in Less than 60 Seconds. In: Kim, S., Yung, M., Lee, H.-W. (eds.) WISA 2007. LNCS, vol. 4867, pp. 188-202. Springer, Heidelberg (2007)

12. Vaudenay, S., Vuagnoux, M.: Passive-Only Key Recovery Attacks on RC4. In: Adams, C., Miri, A., Wiener, M. (eds.) SAC 2007. LNCS, vol. 4876, pp. 344-359. Springer, Heidelberg (2007) 